Enhancing Postgraduate Supervision through a process of conversational inquiry

Dorothy Spiller, Giselle Byrnes and Pip Bruce Ferguson The University of Waikato, Hamilton, New Zealand

Submitted to HERD; presented at Postgraduate Supervision Conversation, Stellenbosch, April 2011 


\title{
Enhancing Postgraduate Supervision through a process of conversational inquiry
}

\begin{abstract}
This paper outlines and begins to evaluate a process to build a critical and reflective community of postgraduate supervisors who can develop their supervision practice through reflective conversations, with the sharing of best practice and reference to research-based evidence. In 2009, the initiative of the Postgraduate Supervisors' Conversations was set up through the collaboration of the Pro-Vice Chancellor (Postgraduate) and the Teaching Development Unit at the University of Waikato, New Zealand. We designed this initiative to complement the compulsory workshops for postgraduate supervisors that are intended to provide foundation skills. We aimed to create a professional development opportunity that could enhance supervisors' capacity to manage the ongoing interpersonal and academic complexity of the supervision process as well as its dynamic character. This paper outlines the rationale for the Postgraduate Supervisors' Conversations, describes its implementation and discusses the implications of an initial evaluative focus group discussion with attendees.
\end{abstract}

\section{Keywords:}

Postgraduate supervision

Postgraduate research

Reflective community

Conversation

Conversational inquiry

Professional development 


\section{Enhancing Postgraduate Supervision through a process of conversational inquiry}

\section{Introduction}

The effort to improve the quality of postgraduate supervision and student research outcomes needs to be understood within the context of significant changes in the tertiary sector. In the last two decades, New Zealand universities, like their counterparts in other western countries, have been under increasing pressure to provide evidence of accountability and quality in all areas. In the area of research, New Zealand introduced the Performance-Based Research Funding (PBRF) process in 2003, which has intensified the obligation on academics to produce and document their research outputs, research collaborations and contributions to building capacity and capability in their fields of research (TEC 2009). Allied with this latter goal is the intention to improve postgraduate research outcomes, along with increasing rates of student retention and completion. One element in the PBRF model (25\%) is specifically associated with the number of completing postgraduate research students at each tertiary institution. ${ }^{1}$ Simultaneously, the government has been reducing its funding contribution to universities, compelling universities to find revenue from other sources, such as income-generating externally funded research contracts. Together, these trends have established immediate external exigencies to enhance the research culture of the university and to provide a high quality learning experience as well as successful outcomes for postgraduate research students. Correspondingly, the University of Waikato has committed itself to realising these goals. 
When the Pro Vice-Chancellor (Postgraduate) was appointed in 2008, she wished to explore and implement new strategies to enhance the quality of postgraduate research and supervision. This is the institutional and strategic background for the genesis and development of the Postgraduate Supervisors' Conversations initiative.

\section{Literature Review}

The changing higher education context and implications for supervision

The changes in the higher education environment over the past two decades have been widely recognised and documented (for example, Camblin and Steger 2000, Pearson and Brew 2002, Akerlind 2005, Knight, Tait and Yorke 2006). For the purposes of this paper, however, only those changes that have a direct relevance for postgraduate supervision are briefly highlighted.

One common theme running through this literature is that in Western societies the financial contribution made by governments to university education has progressively diminished (Ramsden 1998, Mouwen 2000, Fullan and Scott 2009). In Australia, for example, between 1995 and 2005, public funding in higher education declined from $64.8 \%$ to $47.8 \%$ (Fullan and Scott 2009). ${ }^{2}$ In this tight fiscal environment, all universities have been compelled to seek other sources of income, including external research income. A further associated consequence has been the vigorous pursuit of international students who pay full cost (unsubsidised) fees. From a postgraduate supervision perspective, the increased diversity of the postgraduate student cohort brings additional complexity into the supervision process (Owens 2007). These complexities include 
recognising and respecting different cultural values and backgrounds, language and writing issues, and differences in prior learning expectations, history and approaches to learning (Owens 2007). Changes to the ways in which higher education is funded have also influenced students' expectations about the quality of their teaching and learning experience. Students who are paying high fees tend to be making more exacting demands from academics than in the past; contemporary students have been bred (and fed) on the notion of the market-driven university model in which they are consumers and they (quite rightly) expect good value for money (Davies, Hides and Casey 2001, Mora 2001, Fullan and Scott 2009).

Alongside the financial cutbacks, central government and external stakeholders are demanding greater accountability from all higher education institutions. Consequently, New Zealand universities are expected to provide evidence of the quality of their teaching and research in a range of ways. The quality of the postgraduate research experience is reflected in the PBRF exercise, through university academic audits and the Australasian Survey of Student Engagement. ${ }^{3}$ However, an increased climate of accountability not only prioritises the quality of the learning experience, but also the quantity and volume of student completions; worldwide there is pressure on higher education institutions to achieve the timely completion of increased numbers of research degree (McWilliam and Taylor 2001, Pearson and Brew 2002, Brew and Peseta 2004). The process and outcome of the relationship between supervisor and students have, therefore, been catapulted into the public domain and come under intense scrutiny (Wisker 2005). 


\section{Inherent complexity of supervision process}

Increased external scrutiny and the pressures described above mean that universities can not allow the quality and development of postgraduate supervision to be simply a matter of individual choice or chance. Instead, they need to systematically address themselves to enhancing the quality of supervision, maintaining high standards and achieving higher and timely completion rates. In addition to the external forces that make postgraduate supervision education imperative, there is a need to establish supervision development processes that can cater for the inherently complex and dynamic nature of the postgraduate supervision process (Smyth and Maxwell 2008). Smyth and Maxwell (2008) point to some of the ways in which the supervision process involves a unique blend of elements that co-exist in a dynamic way and that may, at times, be difficult to anticipate, recognise and manage. For example, they discuss the way that the control of and responsibility for the process shifts between supervisor and student, the importance of maintaining the delicate balance between process and achieving a finished product, and the distinguishing feature of higher degree research - the creation of new knowledge. The fluidity and complexity of the supervisory relationship is further intensified by the increasingly diverse nature of the postgraduate student population in terms of culture, gender, age and experience (Wisker 2005). ${ }^{4}$ Linear training modules often do not align well with this complexity that is simultaneously relational, process and product-oriented, organisational and intellectual. Furthermore, a standardised training experience cannot accommodate distinctive disciplinary differences, multiple student and supervisor learning approaches and potentially negative academic perceptions about imposed 
professional development schemes (Wisker 2005). As noted by Pearson and Brew, 'supervisor development in the new context of higher education has to focus on supervisors becoming adaptable. Being locked into one model and set of behaviours is no longer acceptable' (Pearson and Brew 2002, 143).

Inevitably, designing professional development systems of support and opportunities for university postgraduate supervisors also needs to recognise the strong vein of discomfort that many academics may have about teaching-related development programmes more generally (Wisker 2005). The traditional privacy of the supervisor-student relationship may further accentuate feelings of resistance be perceived as challenging what is often cited as 'academic freedom', and encourage perceptions of supervision training simply as an institutional imposition.

\section{Evolving a different approach to the development of postgraduate supervision}

The issues outlined above have been recognised and discussed by a number of scholars (Brew and Peseta 2004, Wisker 2005, Smyth and Maxwell 2008). In her introduction to her book on supervision, Wisker outlines the range of views that informed her approach to its design (Wisker 2005). Her comments recognise the complexity of the enterprise as well as the need to accommodate academics' sensibilities and preferences in relation to professional and pedagogical learning:

It has been absolutely crucial that the book should engage readers in dialogue between research and experience, between supervisor and supervisor, supervisor 
and student. . . . It could not succeed if it tried to talk down to or 'train' supervisors. Instead this book encourages reflection, dialogue and an exchange of good practice. Developmental suggestions are built upon and out of these practices (Wisker 2005, 1).

A number of the terms emphasised by Wisker (2005) are core to the design of the Postgraduate Supervisors' Conversations discussed in this paper. In particular, the notion of reflective dialogue drawing on research and shared experience, which is a central element of our conversational model.

A different kind of response to the complexity of the supervision process outlined above is the development of a research matrix as a tool for supervisors to use with students through the supervision process (Smyth and Maxwell 2008). The matrix is co-constructed by the supervisor and the student as the research process develops, so that it is not a schematic tool, but has the potential to cater for the changing rhythms and requirements of the supervision experience. Smyth and Maxwell argue that the use of the matrix can simultaneously enable thoughtful reflection on process, as well as facilitate the attainment of the final product. The need to maintain a delicate balance between these two imperatives, as outlined in Smyth and Maxwell's study, was also a fundamental consideration for us in setting up the Supervisors' Conversations.

In addition to promoting development through dialogue and creating learning opportunities that could accommodate different needs and contexts, we recognised that 
academics would particularly appreciate flexibility and choice in terms of the aspects of supervision they focussed on and when they chose to do so. There are a number of models of professional development for supervisors which are similarly flexible. At the University of Sydney, for example, flexibility and choice is built into the online Postgraduate Supervision Development Programme which is complemented by face-to face workshops and other resources. As Brew and Peseta explain, 'the Programme [at Sydney] provides opportunities for supervisors to study when and where they wish and to choose activities, learning goals and a level of involvement to suit their particular needs and interests' (Brew and Peseta 2004, 6). Another noteworthy and more recent addition to the Sydney Programme is the 'Recognition Model' in which supervisors who have undertaken the Programme develop online case studies of their practice. This innovation enables providers to evaluate the effectiveness of engagement with the programme and also offers a further developmental resource for all supervisors.

\section{Conversations and community}

Bearing in mind all of these considerations, we opted to build a supervisors' community which we hoped would become a critically responsive (and reflexive) learning space in which participants could enhance their own supervisory practice through dialogue, reflection, and the sharing of narratives - underpinned by and with reference to recent research. We also believed that we needed to meet in a space that felt hospitable, encouraged a high level of trust and participation and was one in which academics could be free to come and go, depending on their availability, workload and the relevance of the particular topic under discussion. Moreover, we also wanted the topics for discussion to 
be generated by the participants themselves and thus to 'chart the course' for the remainder of the conversational series. We believed that the sharing of experiences would enable participants to articulate and then reflect on what they were actually doing, instead of instructing them in particular models of supervisory behaviour or paradigms of the supervision process. Finally, we hoped that building on our collective and shared experience would locate 'discussion in the practice of supervision and the behaviour of participants, ensuring that their learning is situated in their particular research contexts' (Pearson and Brew 2002, 139).

Writing in a management context, Ford and Ford (1995), argue for the benefits of conversation as a tool for organizational change. We chose the 'conversational model' as one which could draw on the needs and experiences of participants, but would also develop the culture around supervision within the University to meet institutional goals. The approach we adopted, of building a conversational community, also incorporated a number of other developmental strategies which have been demonstrated as being compatible within the current context of academia; taking into consideration multiple institutional imperatives and the dispositional tendencies of academics. Conversational communities have been shown to provide situated learning opportunities, enable selfauthorship, and draw on well-established models of reflection and appreciative inquiry. They also have the capacity to create a community of trust in which people can voice vulnerabilities and uncertainties and open them to the group for collective problemsolving. At the same time, the conversational context allows academics to protect the sense of autonomy that remains a core belief for many academics (Akerlind 2005). 
An important caveat regarding the use of conversations for academic development is raised by Nelson, Deuel, Slavit and Kennedy. While the context of their study was a collaborative inquiry group of teachers in secondary school setting, they nonetheless highlight a number of highly pertinent questions about translating conversational inquiry into effective changes in practice. In particular, they distinguish between 'congenial conversations' and 'collegial conversations' (Nelson et al. 2010). They argue that 'congenial' conversations, in which the need to avoid conflict is at a premium, cannot bring about change, whereas 'collegial conversations' can be genuinely transformative. Furthermore, they emphasise the importance of developing trust among participants as an absolute pre-requisite if a detailed and honest examination of practice is going to occur.

\section{The evolution of the Postgraduate Supervisors' Conversations at the University of Waikato, New Zealand}

The Postgraduate Supervisors' Conversations at the University of Waikato offers one model of professional development for supervisors through conversational inquiry. The initiative evolved out of discussions in 2008 between the recently-appointed Pro ViceChancellor (Postgraduate) and members of the Teaching Development Unit. At this stage, the University required all new supervisors to attend supervisors' training workshops and to serve an apprenticeship as a member of a supervisory panel or committee before assuming the role of chief supervisor. ${ }^{5}$ These provisions provide a degree of quality assurance in postgraduate supervision. However, we believed that since these workshops focus largely on the role and expectations of the supervisor as well as 
what might be termed 'institutional compliance', there was a need for another complementary initiative addressed towards building a consistent and sustainable quality of professional development for postgraduate supervisors and thus supporting enhanced supervision outcomes for postgraduate students.

\section{The goals of the University}

The University of Waikato, New Zealand, has committed to a number of high-level strategies for the period 2010-13. The present and future strategic direction of the University is underpinned and supported by a number of key goals related to the different aspects of the University's performance. As a research-led institution, staff and student research is given high priority in these goals. In general terms, these include staff and student research being nationally and internationally recognised, the importance of sustaining a creative and dynamic research culture, and the recognition of staff research achievements. Most significantly in this context, the University has also committed to growing the proportion of research postgraduate students and enhancing research postgraduate outcomes - both in terms of quality and quantity.

In our preparatory discussions, we perceived a need to address both the broader goals and institutional imperatives to sustain a dynamic research culture, and the more specific need to improve postgraduate research outcomes and experiences. We saw a conversational forum as potentially creating a lively and reflective community of supervisors who would enhance both their pedagogical and personal understanding of the supervisory role, as well as enhance their own (and others') practice through sharing and discussion. Our 
hope was that such a community would also gradually infuse the broader University community with a deeper understanding of and commitment to quality supervision practice. Our long term goal was, therefore, to complement the mandatory supervisor training with cultural change around postgraduate supervision and encourage and maintain the habit of reflective inquiry around supervision and teaching practice. In these ways, the University could be more effectively equipped to increase the proportion of postgraduate students and enhance postgraduate research outcomes.

\section{Issues to address}

In addition to these strategic imperatives, we believed that foundational training sessions and the dominant supervision 'apprenticeship model' were, in themselves, not enough to develop critical awareness of the complex challenges of the supervisory relationship and process and to support supervisors in managing supervision. Furthermore, there were few mechanisms in place to meet the ongoing professional development needs of more experienced supervisors. We suspected that encouraging the development of a community of reflective practitioners could be a way of generating awareness of both particular and more generic challenges that supervisors face. We also felt that by inviting supervisors to engage with the tools of a conversational forum, supported by researchbased literature and a supportive collegial network, they would be better able to identify and manage these challenges. The work of the Pro Vice-Chancellor (Postgraduate) and one-on-one contact with postgraduate supervisors and postgraduate students had alerted us to many issues that could be articulated and explored in a more searching, comprehensive and sustained way than is possible in a training session. The issues we 
initially identified included the power dynamics inherent in the supervision relationship, cultural and cross-cultural issues, the boundaries of the supervisor's role and interpersonal matters. We chose the approach of a conversational forum to articulate and explore these (and other) issues and then to consider practical options to manage these challenges, in the best interests of both supervisors and students.

The selection of a process of conversational inquiry

As educational developers over a period of more than twenty years, we have an immediate knowledge of many of the reasons why many academics tend to resist professional development opportunities. In part, resistance among university academics relates to a reluctance to engage in professional development in a culture where academics have traditionally been autonomous and self-regulating (Ramsden 1998). In this cultural context, professional development may be easily dismissed and seen simply as an imposed institutional imperative. Furthermore, there has been a powerful and longcherished assumption among academics that a sophisticated and rigorous command of an academic discipline automatically translates into competent teaching as well as supervisory practice. Professional development around teaching (and supervision) is correspondingly often perceived as unnecessary and conducted by people who do not understand the requirements, expectations and vocabulary of specific disciplines and fields of study. The views that we have frequently encountered in relation to a resistance to professional development for teaching could be even more pronounced in relation to postgraduate supervision. As the supervision process requires a sophisticated researchbased disciplinary background, it is fairly natural for supervisors to assume that they are 
uniquely well-qualified to undertake the activity of supervision. Furthermore-depending on the discipline or field of research — postgraduate supervision sits somewhere between research and teaching in terms of definition and this uncertainty (or 'fuzziness') can lead academics to disregard or underplay the pedagogical aspects of the supervision process. ${ }^{6}$ The intensely private nature of the supervision relationship may subliminally diminish any sense of accountability and may also lead academics to see external guidance as an intrusion into what has traditionally been considered a private space. Finally, many supervisors also tend to be senior longstanding academics, many of whom may have never undertaken any kind of formal professional development and may consequently see it as anathema in the context of their career.

In our planning discussions, we decided that a conversational model would help to ameliorate a number of these reservations. The idea of 'supervisors' conversations' signals an informal gathering of like-minded peers, rather than an institutional or obligatory training session. It thus corresponds well with academics' preference for selfregulation and communicates the notion that this is their own space. More positively, many academics might welcome the opportunity to meet and converse with colleagues across the campus. We believed that a hospitable and comfortable environment would help to build an atmosphere of trust and encourage supervisors to examine and discuss concerns that had previously been kept private-or perhaps only discussed with close colleagues. We therefore chose a venue at the WEL Trust Academy of Performing Arts, and were supported by the University in this regard. ${ }^{7}$ Fortunately for our purposes of creating a 'retreat-type space', the building is set among greenery on the edge of the 
Hamilton campus and overlooks a tree-lined lake. We also decided very early on that we would provide a catered lunch for the meetings, both to welcome and nourish our colleagues, but also to allow us to schedule the meetings over a two-hour period in the middle of the day.

In addition, we (and other colleagues) had already experimented in a number of other contexts with the conversational inquiry group model. Most notably this had been in place since 2008 through the establishment of regular conversational forums for departmental leaders and managers in the University. ${ }^{8}$ These are regular lunchtime gatherings at which academic and professional general staff leaders have exchanged views around various themes and have contributed to changes in institutional norms and policies. Further, in the Postgraduate Certificate in Tertiary Teaching, facilitated by the Teaching Development Unit, much of the learning occurs in the context of individual conversations. As teachers on that programme, we have found that the approach fosters the development of habits of inquiry and interrogation around teaching that academics continue to use and develop long after they have completed the qualification. Across the University, we have also initiated a number of other teaching conversation sites, such as the Teaching Network and conversations facilitated by teaching advocates in a range of different disciplines. Institutionally, our hope is that we can complement and support the institutional goals for improvement by promoting and supporting change 'on the ground'. We believe that multiple conversational sites which belong primarily to the academics themselves can provide the most effective way of bringing about the kinds of cultural 
change necessary to transform institutional goals into reality. This was the immediate context into which we initiated the Postgraduate Supervisors' Conversations.

\section{Description of the initiative}

The Postgraduate Supervisors' Conversations have been held at six-weekly intervals during 2009 and 2010. The meetings are structured around a particular theme, selected by the supervisors in advance of the session. Frequently, supervisors suggest future topics on the basis of matters that have generated lively discussion and interest in an earlier session. We believe it is critical that the supervisors determine the choice of theme as this helps to promote and enhance their ownership of the Conversations. The format we have adopted for the Conversations is relatively simple. Attendees register in advance and the Teaching Development Unit organises the catering, room booking and attendee reminders. Members of the Teaching Development Unit and the Pro Vice-Chancellor (Postgraduate) oversee and share the hospitality and facilitation of the Conversations. On arrival, supervisors usually begin with lunch and a brief period of mingling. In those meetings where there have been invited panel members, they are asked to set the scene for the ensuing discussion by briefly addressing the theme from a particular disciplinary perspective. We suggested to speakers that they present in a 5-10 minute timeframe and asked them to keep the comments relatively informal (and, where appropriate, anonymous in terms of mentioning names). We emphasise the brevity of the speakers' presentation as we recognise the risk of falling (perhaps inadvertently) into a scenario whereby information is presented by authoritative 'experts' which would run counter to the idea of building a learning community in which peers evolve their supervision pedagogy together. Similarly, we have made a point of celebrating our local supervision 
knowledge and best practice: the majority of presenters are University of Waikato staff members. From time to time, we have invited people from outside the institution to contribute to the panel discussion and thus provide a fresh perspective or to draw on their particular experience in the area of postgraduate supervision. In one instance, for example, (with reference to the supervision of international students) the panel comprised currently enrolled international doctoral students at the University. The themes canvassed to date in the meetings have included cross-cultural supervision, co-publishing with students, negotiating understandings between supervisors and students and written feedback on students' draft writing (see Appendix One for a list of topics).

\section{Evaluation Methods}

Through 2009 and 2010, attendance at the Conversations has been sustained at a good level (about 30 per session). ${ }^{9}$ Discussions have been lively and the ongoing informal feedback from attendees has been extremely positive. We recognised, early in the second year, that we needed to embark on a more systematic evaluation process in order to gauge the effectiveness of the Conversations and also to inform the way we plan, refine and develop future Conversations. To this end, we sought approval from the University of Waikato Human Research Ethics Committee to initiate a formal action research investigation of the Postgraduate Supervisors' Conversations.

In the application for ethical approval, we outlined our proposal to run a final end-of-year meeting of the Supervisors' Conversations as a focus group discussion in order to evaluate the meetings to date and to look ahead to future topics for conversation. The 
Application was approved by the University Human Research Ethics Committee and the final meeting session was advertised in the Official Circular of the University. We invited all of those supervisors who had attended the previous two years' sessions to come and share their views; or, if they were unable to attend, we invited them to provide feedback via email. We formally explained the purposes of the session and informed supervisors of the proposed research project (Official Circular 12 November 2010).

For the Focus group, we designed a short PowerPoint presentation explaining the research goals and the uses of the findings. We also provided a set of broad questions to act as prompts for the discussion. The questions were as follows:

How useful have you found these discussions/presentations?

What has been of particular benefit?

Please nominate the three most valuable aspects of these meetings.

What have you found most challenging?

Please nominate the top three most challenging aspects of these meetings?

In what ways have these discussions encouraged you to reflect on your own supervision practice?

What would you be interested in seeing on the list of topics for 2011 ?

After the presentation, we invited participants to discuss the questions in the groups in which they happened to be seated and then a TDU member facilitated the general discussion which followed. We emphasised that our goal was to open up the discussion 
and capture a range of perspectives, rather than attempting to reach any sort of 'group consensus.'

\section{Findings}

Twenty-two supervisors attended the final evaluative session and we received additional feedback via email. The findings in response to the key questions were as follows:

\section{Usefulness}

There were eight recorded responses in this category. Of these, six used emphatic reinforcers to endorse their positive views, such as 'very useful', 'absolutely fabulous' and 'excellent'. The other two responses were positive without being obviously and overtly enthusiastic.

\section{Benefits}

Of the 24 recorded comments here, eleven used a range of words and phrases that highlighted the value of connecting with others, networking and learning from each other and building a community of support. Some examples of this kind of feedback included:

Talking with people in my faculty that I don't see often; talking with and listening to those from other disciplines.

Listening, sharing and understanding other people's processes and concerns. 
Gaining insight from practices in other disciplines.

Sharing different perspectives and experiences.

Networking [3 respondents].

All of this lifts supervision from an ISOLATED experience; start of a community [2 respondents; emphasis in the original].

Providing a voice for issues; the group can seek answers.

Alongside this endorsement of the collective learning opportunity, specific comments pointed to more precise observations about the intellectual, emotional and practical benefits of learning in this way. These observations include terms such as 'making tacit knowledge explicit', 'reflection space', 'reassurance', 'big picture', 'getting to know about University rules/regulations, expectations/standards', and 'the role of the chief supervisor'. Five comments drew attention to the high quality of the conversational environment. Three of these responses offered enthusiastic comments about the food, one praising the location and one approving the open and honest conversational environment. This participant observed:

Liked not recording it so felt like everyone was open and frank. People who came wanted to talk and share so it is a self-selecting group who are open to reflecting. 
One participant raised the problem that the benefits of the Conversations were not reaching those who may have perhaps most needed it; that is, those who did not attend the sessions but who were perceived, by their colleagues, as needing to be present. We emphasise here that these meetings were (and remain) voluntary.

\section{Challenges}

Of the 17 recorded responses to this question, ten common responses related to participation from two main perspectives. The first of these relates to the already expressed concern regarding the limited representation of supervisors at the Conversations (four). These comments include:

More participation of [relevant committee] members.

Those who should attend do not.

Uneven distribution of participating Schools [i.e., academic units].

The second set of comments about participation relate to the view that there could still be more widespread participation in the discussion by conversation attendees and that more time and space for open discussion was needed. Comments along these lines included the following:

Wanted to hear from even more people-sometimes not enough time (although two hours is about the right length). 
More input from emerging supervisory group.

If a discussion becomes dominated by a person/faculty-but this didn't happen often.

Would be good to have more space for group discussions. I think we are only beginning to develop GROUP TRUST [emphasis in the original].

Another participation-related comment was a preference for 'local' rather than 'outside' panel and guest speakers, a point associated with a preference for the conversational model. One attendee acknowledged that the outsiders provided 'good information', but 'they were more like presentations than conversations.'

Other challenges identified by participants related to more specific issues or topics that had been discussed. In this respect, four comments related to the challenges (and joys!) of supervising across cultures or languages and a comment was raised about the challenge of understanding research paradigms and processes in different disciplines. On another level, one participant found it challenging to speak as an individual amidst certain dominant practices, or the individual voice 'versus variances and behaviours in the department.'

In what ways have these discussions encouraged you to reflect on your own supervision practice?

Of the 13 comments recorded in this section, six indicated that the Conversations play a role as something of a touchstone for examining supervisors' own practice, or functions 
as a place where they can test out concerns around their supervision experience and understanding. Words used in this regard include 'confirming', 'clarify', 'reflecting back', 'reflect on concern issues and competencies', 'see what others do, especially when it's relevant to one's own work', 'a debriefing venue', and 'checking my own ideas and experience against others'.

Other comments identified more specific areas of supervision practice that the participating supervisors have been alerted to through the conversational process. These include becoming more 'conscious of teaching loads', '[the] balance of research, supervision and lecturing', 'co-publishing with students', working with students in 'developing the [research] proposals', ideas about 'when to push and when to withdraw', and different approaches to clarifying and establishing student and supervisor expectations in the supervision relationship.

A significant thread of comment was the need for the institution to formally recognise attendance at the Supervisors' Conversations as part of staff professional development.

\section{Future topics}

There were 33 suggestions recorded in this section, covering a wide range of topics. Five suggestions related to the idea of placing more emphasis on the student perspective of postgraduate supervision. These included observations such as increasing '[the] student voice', calling for more 'understanding [of] the context of students' and 'students' views', and asking 'are students getting what they need?' Four suggestions expressed a 
desire for more reflection on working with students from different cultures, and a further four related to various aspects of the relationship between supervisor and students. Other ideas highlighted more specific concerns, such as the role of the supervisor in 'producing academic writing', 'cross-discipline supervision', 'supervision workload', 'ensuring the quality of a student thesis', and 'supervising colleagues'.

\section{Discussion and conclusion}

Our experience of supporting and facilitating the Supervisors' Conversations over a period of two years, together with the findings from our focus group research, provide us with a starting point for evaluating the Conversations and their efficacy-both for individual staff members and for the institution. Such feedback has also been invaluable to assist us in our future planning. In the following section, we evaluate what we have achieved against key criteria. These are the external context and the goals of the University, the rationale for the design and implementation of the initiative, and the perspectives we have highlighted from the literature.

\section{The external context and the goals of the university}

In terms of the University's strategic intentions, our experience and feedback suggests that the Conversations are contributing to our collective effort to foster and support a creative and dynamic research culture. Attendance has been sustained at a healthy number, the discussion has been lively and constructive and there has been a considerable and genuine sharing of views about supervisory practice. Focus group feedback suggests that participants similarly value the shared exchange of ideas and practices and that these 
have provided a touchstone for assessing, and evaluating individual practice. These comments suggest a heightened attention to and reflection upon 'the business' of postgraduate supervision, which can in turn enhance the research culture and has real and lasting potential to improve practice as well as postgraduate research student outcomes.

At this stage, it would be reasonable to suggest that the Postgraduate Supervisors' Conversations have enlivened and stimulated the culture around postgraduate supervision and research, but it is difficult to evaluate their contribution to actual supervisor practices and students' experiences of supervision. Additionally, we are not yet in a position to claim that we have improved the University's capacity to respond to external demands around postgraduate learning (McWilliam and Taylor 2001, Pearson and Brew 2002, Brew and Peseta 2004). This is partly because the format of the Conversations has been deliberately designed as open-ended and participatory and does not, therefore, fit neatly into a training model. However, despite these limitations, our findings point to a need to further embed a process by which the Conversations enhance supervision practices (Brew and Peseta 2004). The model presented by Brew and Peseta, where online case studies of supervision practices are shared by participants on their Supervision Programme, may provide a way forward. Not only does this strategy provide a way of sharing practices more widely, but is also provides evidence for recognising and rewarding positive supervision practice.

The rationale for the design and implementation of the initiative 
We chose the model based on community and conversations to provide a dynamic forum in which academics have the opportunity to explore, articulate, assess and redefine the complexities inherent in the supervision process. Our focus group findings reveal that the two most valued benefits of the Conversations are, first, the notion of a network or community, and second, the opportunity to learn from each other. The findings strongly endorse our starting point that the Conversations could be a place in which academics like to learn and that they would enjoy the chance to learn from each other in a peer-topeer situation. Participants' appreciation of the relaxed environment, the hospitality and the conversational sharing indicate that we have made some progress in establishing an open and inviting community, and moreover, one that is different from the context of a formal training session. On the basis of our findings and experience, it is difficult to estimate the extent to which our Conversations went beyond 'congenial' discussions towards genuine 'collegial discussions' that were truly transformative (Nelson et al. 2010). Comments indicate that participants felt challenged to revisit their own practice, but it is difficult to gauge the extent of this personal and professional stocktaking. In this respect, perhaps the best way forward is to continue to work on building trust and respect in the conversational community so that it can enable genuine rigorous debate and selfevaluation.

The two most commonly reported challenges articulated by participants related to nonparticipation. The first concerns the view that many other supervisors should be attending the sessions and that the supervisors who regularly attended should be more widely representative of the University's supervisor community. As mentioned in the previous 
section, one strategy for broadening the base of the Conversations may be the development of some form of complementary online module (Brew and Peseta 2004). At the same time, the wish to share the benefits more widely is a strong indicator of how positively attendees view the Conversations. The second recurring thread in the feedback around participation concerned the expressed desire to increase the amount of conversational space and to limit the time devoted to formal presentations. This feedback endorses our original view that academics like to feel that they have authorship of their own professional development and learning. Attendees' comments in this respect also have implications for planning and refinement of the forum for future years. Modifications to explore could include a more precise briefing to panel members and variation to the panel format in terms of the initial conversation stimulus. For example, one idea could be to select a reading or reported case study that colleagues read prior to the session that then provides the starting point for discussion. The range of specific benefits that participants reported and the comprehensive list of ideas for future conversations - particularly with regard to matters of managing relationships - suggests that the Conversations have begun to unlock the inherent complexity and dynamic character of the supervision process (Wisker 2005, Smyth and Maxwell 2008) while also becoming part of the fabric of our institutional culture.

In terms of our goals for the initiative as well as the institutional goals, the transference of conversational learning to supervision practice is crucial. In the focus group we asked respondents about the ways in which the Conversations had encouraged them to reflect on their own practice. Six out of 13 responses to this question affirmed that the 
Conversations had worked in this way, using terms such as 'reflect', 'checking', 'clarify', and 'debriefing'. While these attendees are a self-selected and small group, their comments nonetheless indicated the value of collegial conversation as a stimulus for reflection on their own practice. However, there was little evidence in the findings of actual changes to supervision practices. While we did not specifically plan to monitor such changes and did not ask this question directly in the focus group, we will need to consider how to promote and evaluate practical enhancements in future iterations of the Conversations. Such planning should take us further towards responding to both external and institutional demands for improved postgraduate outcomes, as well as enhancing the quality of the supervision experience, both for students and supervisors alike. At the same time, we will have to find strategies for promoting and evaluating practical changes in supervision practices that do not undermine the concept of an open, collegial forum in which colleagues are comfortable to share vulnerabilities, difficulties and uncertainties. Thus far we believe that we have begun the journey of building a community in which conversational interchange can happen in an intellectually charged atmosphere that, at the same time, feels safe. In our next iteration of the Supervisors' Conversations, we need to consider strategies that will help translate conversational inquiry and reflection into enhanced supervision practice. 


\section{Appendix One: The University of Waikato Postgraduate Supervisors'}

\section{Conversations 2009 and 2010}

2009

15 July: Best Practice in Postgraduate Supervision

23 September: 'Hot Topics' around supervision

4 November: Interdisciplinary supervision: composition of panels, conversations across disciplinary boundaries, opportunities and risks

2010

23 April: Cross-cultural literacy

4 June: Co-publishing with students

21 July: Negotiating the terrain of supervision ('MOU' between students and supervisors)

29 September: Best practice for encouraging and supporting Maori doctoral students

3 November: Feedback on student draft writing

5 December: Reflections to date

\footnotetext{
1 The PBRF in turn supports the Tertiary Education Strategy, which outlines the New Zealand Government's priorities for tertiary education sector, by encouraging an integrated, specialised tertiary education sector and developing research capability. The New Zealand Government sees the tertiary education system as a key national asset, which enriches New Zealanders' lives, increases their employment opportunities and helps to build a productive skill base to drive economic growth. See further http://www.minedu.govt.nz/theMinistry/PolicyAndStrategy/TertiaryEducationStrategy.aspx (accessed 25 January 2011).

2 In New Zealand, government (public) expenditure on tertiary education has increased by $53 \%$ from 1999/2000 to 2010, http://www.educationcounts.govt.nz/indicators/resources/2047 (accessed 26 January
} 
2011). However, the increase in student numbers at all New Zealand universities, combined with the increase in university costs during this period, actually represents a decrease in real terms from 2007 to 2010. See further 'Government funding fails to keep pace with university costs', 28 September 2010, http://www.nzvcc.ac.nz/node/480 (accessed 26 January 2011).

${ }^{3}$ The Postgraduate Survey of Student Engagement (POSSE), which is part of the Australasian Survey of Student Engagement (AUSSE), see further http://ausse.acer.edu.au/ (accessed 25 January 2011).

${ }^{4}$ Denholm and Evans note that in Australia and New Zealand, the age range of $\mathrm{PhD}$ candidates ranges across fifty years (Denholm and Evans 2007, 2).

5 The Supervisors' Workshops are still offered and are run by the Pro Vice-Chancellor (Postgraduate), the Postgraduate Studies Committee and the Postgraduate Studies Office. The University of Waikato has a Supervisors' Register and a Chief Supervisors' Register; certain pre-requisites must be met before a staff member is approved to join one or both registers.

${ }^{6}$ Dr Barbara Grant uses the metaphor of 'walking on a rackety bridge' to refer to the supervisory relationship (Grant 2010, 10-12).

7 The venue for the Supervisors' Conversations is one of the most beautiful buildings on the University of Waikato (Hamilton) campus. See http://www.waikato.ac.nz/academy/academy.shtml (accessed 26 January 2011).

${ }^{8} \mathrm{We}$ are indebted here to the conversational model 'piloted' in both the Chairpersons' Forums and the Managers' Forums, led by our Human Resources Division, and in particular, Anna Bounds, Assistant ViceChancellor (Executive) and Mike Bell, Human Resources Division, The University of Waikato.

${ }^{9}$ The University of Waikato has approximately 600 academic staff members, not all of whom supervise at postgraduate level. 


\section{References}

Akerland, G.S. 2005. Academic growth and development: How do university academics experience it? Higher Education :501-32.

Brew, A. and T. Peseta. 2004. Changing postgraduate supervision practice: a programme to encourage learning through reflection and feedback. Innovations in Education and Teaching International 41 (1):5-22.

Camblin, L.D. and J.A.Steger. 2000. Rethinking faculty development. Higher Education 39:1-18.

Davies, J.M., T. Hides, and S. Casey. 2001. Leadership in higher education. Total Quality Management 12 (7 and 8):1025-30.

Denholm, C. and T. Evans, eds. 2007. Supervising Doctorates Downunder: Keys to Effective Supervision in Australia and New Zealand. Camberwell, Victoria: Acer Press.

Ford, J.D. and L.W. Ford. 1995. The role of conversations in producing intentional change in organization. Academy of Management Review 20 (3):541-70.

Fullan, M. and G. Scott. 2009. Turnaround leadership for higher education. San Francisco: Jossey Bass.

Grant, Barbara. 2010. Closing Metaphor: Supervision as a Rackety Bridge. TDU Talk 7:10-12. https://www.waikato.ac.nz/tdu/pdf/tdutalk/Sep10.pdf (accessed 26 January 2011).

Knight, P., J. Tait, and M. Yorke. 2006. The Professional learning of teachers in higher Education. Studies in Higher Education 31 (3):319-39. 
McWilliam, E., and P.G. Taylor. 2001. Rigorous, rapid and relevant: Doctoral training in new times. In Doctoral education and professional practice: The next generation? ed. B. Green, T W. Maxwell, and P. Shanahan, 229-46. Armidale, NSW: Kardoorair Presss. Mora, J. 2001. Governance and management in the new university. Tertiary Education and Management 7 (2):95-110.

Mouewen, K. 2000. Strategy, structure and culture of the hybrid university. Tertiary Education and Management 6 (1):47-56.

Nelson, T.N., A. Dueul, D. Slavit, and A. Kennedy. 2010. Leading deep conversations in collaborative inquiry groups. The Clearing House 83:175-79.

Owens, R. 2007. Valuing international research candidates. In Supervising Doctorates Downunder: Keys to Effective Supervision in Australia and New Zealand, ed. C. Denholm and T. Evans, 146-54. Camberwell, Victoria: Acer Press.

Pearson, M. and A. Brew. 2002. Research training and supervision development. Studies in Higher Education 27 (2):135-49.

Ramsden, P. 1998. Learning to lead in higher education. London and New York: Routledge.

Smyth, R. and T.W. Maxwell. 2008. The research matrix: An approach to supervision of higher degree research. HERDSA Guide. Milpera, New South Wales: Higher Education Research and Development Society of Australasia.

TEC, see Tertiary Education Commission.

Tertiary Education Commission. 2009. Performance Based Research Funding. http://www.tec.govt.nz/Funding/Fund-finder/Performance-Based-Research-Fund-PBRF-/ (accessed 25 January 2011). 
Wisker, G. 2007. The good supervisor. Basingstoke, Hampshire and New York: Palgrave Macmillan.

University of Waikato. Official Circular. 12 November 2010. 